\title{
Linx
}

Revue des linguistes de l'université Paris X Nanterre

$53 \mid 2005$

Le semi-figement

\section{Figement absolu ou relatif : la notion de degré de figement}

Salah Mejri

\section{CpenEdition}

Journals

Édition électronique

URL : http://journals.openedition.org/linx/283

DOI : $10.4000 / \operatorname{lin} \times .283$

ISSN : 2118-9692

Éditeur

Presses universitaires de Paris Nanterre

\section{Édition imprimée}

Date de publication : 1 décembre 2005

Pagination : 183-196

ISSN : 0246-8743

\section{Référence électronique}

Salah Mejri, «Figement absolu ou relatif : la notion de degré de figement », Linx [En ligne], 53 | 2005,

mis en ligne le 15 février 2011, consulté le 19 avril 2019. URL : http://journals.openedition.org/linx/283 ; DOl : $10.4000 /$ linx.283

Département de Sciences du langage, Université Paris Ouest 


\title{
Figement absolu ou relatif : la notion de degré de figement
}

\author{
Salab Mejri \\ Université de Paris 13 et Laboratoire LLI
}

Il est incontestable qu'actuellement le figement est devenu une dimension fondamentale dans la description des langues. Plusieurs travaux sur la question ont vu le jour à la fin du $\mathrm{XX}^{\mathrm{e}}$ siècle, mais ceux-ci, tout en l'imposant comme un champ d'investigation à part entière dans la recherche linguistique, n'ont pas pour autant réussi à faire émerger une vision unifiée du phénomène. Nous voudrions fournir dans ce qui suit une idée relativement précise sur le statut du figement dans les études linguistiques, préciser des critères permettant de conclure à un figement absolu avant d'aborder la question de son aspect relatif.

\section{Statut du figement dans les études linguistiques}

Outre la dimension terminologique, il y a lieu d'effectuer une évaluation du phénomène à travers les études les plus pertinentes sur la question, tout en focalisant sur la dimension du traitement informatique des séquences figées (SF).

\subsection{Problèmes terminologiques}

Parmi les questions centrales dans l'évaluation épistémologique de tout processus de découverte, il est indéniable que la dimension terminologique sert de repère relativement fiable dans la fixation et la stabilisation des objets de la recherche. 
Or parmi les concepts essentiels de la terminologie figurent les dénominations centrales qui désignent l'objet de la discipline ou de la section revendiquée.

Pour en faire une évaluation qui reflète l'état de la question, nous avons choisi comme repère le dernier dictionnaire de linguistique, celui de Franck Neveu qui a l'ambition de rendre compte de ce qui existe, de « refléter un état de la discipline des sciences du langage observable à partir de son vocabulaire, c'est-à-dire à partir des pratiques terminographiques effectives » $(2004: 6)$. Trois termes renvoient au figement :

- collocation:

une co-occurrence conventionnelle, résultant d'une forte contrainte sémantique de sélection qui se manifeste dans la valence d'une unité lexicale, et qui a pour effet de restreindre la compatibilité des mots avec l'unité en question. La notion sert principalement à décrire les assemblages lexicaux habituels, autrement dit ceux qui sont entérinés par l'usage

- figement:

un ensemble de caractéristiques syntaxiques et sémantiques affectant une unité polylexicale $[\ldots]$ le figement peut n'être que partiel, ce qui suppose des degrés dans le processus (fait divers est plus figé que fait historique). Cela suppose donc également un continuum entre séquences libres et séquences contraintes

- phraséologie:

l'ensemble des unités complexes du lexique qui présentent des degrés variables de figement, qui sont construites dans des contextes spécifiques, et qui sont tenues à cet égard pour caractéristiques d'un type de discours

Comme on le constate, les trois définitions semblent complémentaires. La collocation renvoie à l'unité figée, le figement aux caractéristiques des SF et la phraséologie à la partie figée du lexique. En fait, il n'en est rien : même s'il est vrai que le figement dénomme le processus, la collocation n'est en fait qu'un type particulier de $\mathrm{SF}$, celles dont la polylexicalité est en voie de fixation. Quant à la phraséologie, elle fait partie de la terminologie en usage dans l'Est Européen. L'autonomie des trois termes est appuyée par l'existence de paradigmes parallèles :

.collocation, collocatif, collocationnel...

. figement, figé, expression (séquence) figée...

- phraséologie, phrasème...

S'ajoutent à cet enchevêtrement terminologique toute une série d'autres termes comme idiome, idiotisme, idiomatique, locution, etc. En réalité, cette situation traduit le flottement conceptuel dans ce domaine. Il y une instabilité terminologique qui concerne aussi bien le phénomène que le produit. Pour résumer la situation, on peut dire : 
- que les définitions se recouvrent totalement ou partiellement avec la persistance d'un flou qui traduit les difficultés à cerner le phénomène ;

- qu'il y a des difficultés à isoler clairement ce qui relève de chaque domaine ;

- qu'il est nécessaire de hiérarchiser les trois concepts.

Mais cela ne signifie pas que les travaux sur le figement n'ont abouti à aucun résultat. Au contraire, outre la reconnaissance déjà mentionnée, nous disposons actuellement d'une série d'études qui apportent des éclairages non négligeables sur la question. Nous en fournissons une synthèse.

\subsection{Apport des études portant sur le figement}

Peut-être faudrait-il rappeler, avant de passer en revue les travaux qui ont marqué ce domaine, que nous savons maintenant au moins que :

. $20 \%$ des textes seraient composés de segments répétés (Fiala et al. 1978);

. le nombre d'unités polylexicales dépasse de très loin les unités monolexicales appartenant à la même partie du discours: noms, verbes, adjectifs, adverbes, déterminants, etc. (M. Gross 1982, 1988);

. le figement transcende la répartition en parties du discours et touche des segments phrastiques ou infra-phrastiques sans que ces unités soient pour autant réductibles à une partie du discours (Mejri 1997);

. le figement ne se limite pas au lexique; il a pratiquement le monopole de la grammaticalisation (Mejri 2001).

Indépendamment de telles considérations, les travaux de certains linguistes ont enrichi amplement la réflexion sur la question et ont ainsi contribué à une sorte de balisage général du terrain. Ainsi devons-nous respectivement à M. Gross, Gréciano et Ruwet un ensemble de repères en matière de syntaxe et sémantique qui font actuellement partie des connaissances partagées sur le figement. C'est à M. Gross qu'on doit la découverte de la conformité entre la formation interne des séquences figées et la syntaxe libre, la préconisation qu'il faut intégrer la description des séquences figées dans la description de la syntaxe générale ( $c f$. en particulier La syntaxe de l'adverbe) et qu'il est nécessaire d'élaborer des tests spécifiques aux unités polylexicales décrites.

A Gréciano (1983) nous devons l'introduction de la notion de «polylexicalité » comme fait définitoire des séquences figées, la description de la signification des séquences figées en termes de conceptualisation et l'explication de l'opacité sémantique par l'intervention des mécanismes de la figuration et de l'aréférenciation. Quant à Ruwet (1983), il a réfuté certaines thèses de Chomsky relatives aux séquences figées au moyen de l'analyse de plusieurs contre-exemples, appliqué les transformations courantes aux séquences figées et abouti à la conclusion que les contraintes relevées dans le fonctionnement des séquences figées ne sont pas spécifiques à ce genre de séquences mais partagées par des combinaisons considérées comme libres ( $c f$. le passif 
par exemple). C'est avec G. Gross (1996) et R. Martin (2001) que nous aboutissons à une lecture unifiée du phénomène. Si le premier aborde le figement dans sa globalité, le second privilégie la dimension relative au traitement automatique. G. Gross a établi la première liste de critères généraux permettant de reconnaitre les séquences figées, introduit d'une manière explicite la notion de « degrés de figement » et fourni des tests spécifiques aux séquences réparties selon leur appartenance aux parties du discours concernées. Pour Martin, le figement représente, à côté de la polysémie et de l'inférence, l'une des grandes difficultés pour le traitement automatique. Cette difficulté se situe au niveau de la reconnaissance de l'unité globale et de sa génération ; mais vu sous l'angle des dictionnaires informatisés comme le TLF, le figement peut être considéré comme un grand avantage puisqu'on dispose d'une importante base de données d'unités polylexicales auxquelles sont attribuées des significations bien précises.

L'intérêt porté à cette question se vérifie également grâce aux publications entièrement consacrées au figement. Nous en retenons celles de 2003 : un numéro spécial de Cabiers de lexicologie, un autre de Syntaxe et sémantique et un troisième de Travaux et recherches en linguistique appliquée. Dans la première publication figurent plusieurs tentatives de définition du figement. Selon Mortureux par exemple, le figement serait «une étape dans un processus dont la lexicalisation serait le terme 》 (p. 5-6). Deux idées centrales s'en dégagent également: la complexité du phénomène mérite une démarche capable de rendre compte des multiples facettes des séquences figées ; il est nécessaire de procéder à des descriptions locales très fines impliquant la totalité des aspects du système linguistique (prosodie : le cas des proverbes par exemple, syntaxe, sémantique, dimension pragmatique, etc.). Dans la deuxième publication, où l'on oppose la polysémie à la polylexicalité, on avance l'idée que la structuration du système linguistique se fait sur la base d'une dissymétrie fondamentale entre la pluralité du signifié (la polysémie) et la pluralité du signifiant (le cas de la polylexicalité) : ainsi «la polylexicalité serait aux séquences figées ce que la polysémie est aux unités lexicales simples ». La polylexicalité est présentée comme une caractéristique dont découle l'essentiel des contraintes de fonctionnement syntaxique et sémantique des séquences figées.

Le figement en tant que phénomène général ne touche pas uniquement la langue générale mais également les langues spécialisées. Le dernier ouvrage est consacré aux collocations. Pour les auteurs, les collocations, à la différence des unités phraséologiques, sont des expressions semi-figées binaires, constituées de deux expressions linguistiques (p. 7-8). Appliquant la théorie de Mel'čuk, ils se sont heurtés surtout au caractère opaque de son métalangage (p. 13).

De toute cette littérature se dégage un ensemble d'outils permettant de faire la distinction entre figement absolu et figement relatif.

\section{Le figement absolu}

Nous entendons par «figement absolu » le degré maximal de «fossilisation» d'une formation syntagmatique. Des séquences comme advienne que pourra, fait divers, à bon entendeur salut, au fur et à mesure, etc. en constituent une bonne illustration. Il faut 
reconnaitre que la vision la plus répandue du figement était celle de considérer que les séquences figées l'étaient entièrement. Ce n'est qu'après plusieurs travaux qu'on a réussi à dégager le figement de cette vision. En réalité, le figement absolu ne touche en moyenne qu'une expression sur dix. Pour le savoir, il a fallu forger des critères formels et sémantiques et les classer en fonction de leur pertinence. Nous en fournissons un résumé succinct.

\subsection{Critères formels}

On distingue deux générations de critères formels :

- une première génération de critères appliqués aux séquences figées : l'absence de déterminant devant l'élément nominal de certaines séquences verbales (Gougenheim 1971) : avoir peur, avoir soif, avoir faim;

- une seconde génération: les transformations (M. Gross 1982). Pour un exemple comme casser sa pipe, M. Gross propose un ensemble de tests :

a. la variation de la personne et du nombre du possessif :

$$
\text { Luc et Max ont cassé leur pipe }
$$

b. la variation du mode du verbe :

$$
\begin{aligned}
& \text { Je crains que Max n'ait cassé sa pipe; } \\
& \text { En cassant sa pipe, Max a sauvé ses complices }
\end{aligned}
$$

c. l'emploi d'adverbes de temps :

$$
\text { Max va casser sa pipe dans peu de temps }
$$

d. l'insertion d'éléments entre le verbe et le complément :

$$
\begin{aligned}
& \text { Nous casserons tous notre pipe un jour ; } \\
& \text { Max a bêtement cassé sa pipe }
\end{aligned}
$$

e. la pronominalisation :

$$
\text { Luc a cassé sa pipe et tu la casseras un jour aussi }
$$

f. la substitution d'un verbe synonyme au verbe casser:

*Max a rompu sa pipe

g. la substitution d'un nom synonyme ou proche de pipe:

*Max a cassé son brûle-gueule

h. le non-respect de la coréférentialité du pronom au sujet :

$$
\text { * Max a cassé leur(s) pipe(s) }
$$

i. l'introduction de modifieurs :

* Max a cassé sa vieille pipe 
Salab Mejri

j. le clivage :

*C'est sa pipe que Max a cassée

k. la passivation :

* La pipe a été cassée par Max

1. l'interrogation :

* Qu'a cassé Max? - Sa pipe

m. la relativation :

* La pipe que Max a cassée fascine Luc

Les critères fournis appellent les remarques suivantes :

- les tests ne peuvent pas être conçus en termes de présence ou d'absence d'éléments formels comme c'est le cas pour l'absence du déterminant : avoir peur mais avoir une peur bleue ou avoir une de ces peurs; avoir peur mais avoir la trouille;

- les travaux menés sur la prédication montrent clairement que peur est un prédicat nominal (M. Gross, J. Giry-Schneider et G. Gross) actualisé par le verbe support avoir. Le français, n'ayant pas de verbe monolexical comme *peurer (une langue comme l'arabe dispose de verbes simples pour signifier avoir peur : جاف خa :f, avoir soif: ؟tal عطش avoir faim: [cf. particulièrement l'analyse de G. Gross des deux séquences avoir soif et avoir froid où il montre que dans un cas, il s'agit d'un prédicat nominal et dans l'autre d'une locution verbale (1996: 74-75)] ;

- indépendamment de la catégorie grammaticale des séquences fournies en exemples (des locutions verbales), nous relevons une opposition de nature entre les cinq premiers et le reste des tests appliqués à casser sa pipe. La raison en est que les premiers ne remettent pas en cause l'intégrité conceptuelle de la séquence alors que les derniers y portent atteinte, d'où le défigement qui s'ensuit.

\subsection{Critères sémantiques}

Si la rupture sur le plan formel se voit à travers l'acceptation ou le refus des restructurations syntaxiques de la séquence, les caractéristiques sémantiques se ramènent à deux points :

\subsubsection{Les mécanismes sémantiques à l'oeuvre dans la $S F$}

Il s'agit des mécanismes tropiques qui interviennent dans la structuration sémantique de la SF et qui conduisent selon les cas à des séquences opaques ou transparentes. Dans ce dernier cas, le sens est dit compositionnel (avoir soif); dans le premier, le sens global n'est pas déductible de celui des constituants (tête-de-mort: nom usuel du muflier, dent-de-lion: nom donné au pissenlit, pied-de-veau: nom vulgaire de l'arum) (Mejri 1997 : 322).

L'opacité a été longtemps considérée comme le critère absolu du figement: manger les pissenlits par les racines, prendre le taureau par les cornes, etc. Le résultat, c'est que la 
transparence est de ce fait exclue, ce qui n'empêche pas que le nombre d'unités polylexicales non opaques soit considérable: une heure de grande écoute, un soldat de deuxième classe, une offre publique d'achat, la clause de la nation la plus favorisée, etc. Devant un tel constat et tenant compte de séquences comme briser la glace/la glace brisée, qui acceptent de fonctionner comme les séquences libres en admettant le passif sans que le sens littéral soit nécessairement activé, on a commencé à s'orienter vers une position plus nuancée : l'opacité, tout en étant une caractéristique des séquences figées, n’en est pas pour autant un élément définitoire.

Interviennent également dans le sémantisme des SF les trois notions suivantes : la conceptualisation (Mejri 1998 : 41-48), la globalisation (1998 : 83-93) et la dénomination polylexicale (2000: 609-621).

\subsubsection{Contenu catégoriel et contenu sémantique particulier}

Si les exemples fournis sont des locutions verbales, c'est parce qu'elles font partie des séquences figées qui admettent le plus de manipulations si on applique aux séquences la distinction entre contenu sémantique catégoriel et contenu sémantique particulier. Le contenu catégoriel, tel que nous l'avons défini en 1997 et en 1998, renvoie à l'appartenance de toute unité à une partie du discours, alors que le contenu particulier concerne le contenu sémantique qui se charge de l'intension de l'unité lexicale, ce qui rappelle l'opposition faite par Tesnière entre substantif général (le pronom) et substantif particulier (les substantifs comme livre, cabier, etc.).

L'analyse des deux contenus montre clairement qu'il y a une hiérarchie entre le premier contenu et le second : le contenu catégoriel est hiérarchiquement supérieur au contenu particulier; celui-là conditionne le fonctionnement de celui-ci. Deux cas de figure se présentent : soit la séquence polylexicale est conçue dans le cadre de la même partie du discours (unité auto-entité), soit dans une autre partie du discours (unité hétéro-entité). Nous avons fourni en 2003 (p. 31) le tableau suivant pour montrer comment les séquences figées se répartissent dans le cadre des quatre parties du discours « prédicatives » dans le sens guillaumien du terme :

Parties du disc. N Adj. V Adv.

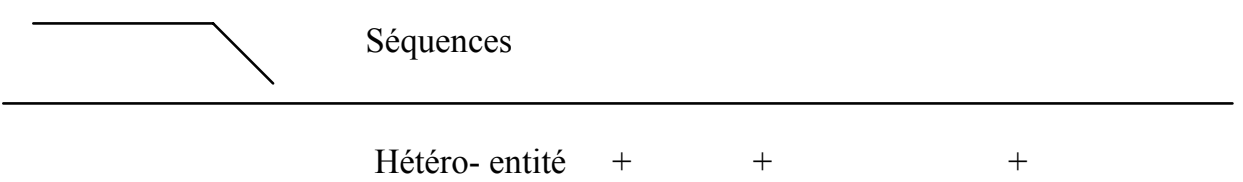

Auto- entité

$(+)++$ 
Salab Mejri

En appliquant les éléments dégagés plus haut aux SF, on peut dire que la SF prototypique qui n'admettrait aucune variation formelle et qui témoignerait d'un figement absolu serait une séquence figée hétéro-entité et complètement opaque.

\section{Le figement relatif}

Cette notion ne peut se concevoir indépendamment de celle de degrés. Si le figement absolu, contrairement à ce qu'on croit, ne concerne qu'une partie des séquences figées, on doit s'orienter du côté d'une approche qui touche au fonctionnement des SF. Les séquences qui rejettent toute variation et qui peuvent être considérées comme relevant d'un figement absolu sont soit des vestiges d'un ancien état de la langue appelés par G. Gross « des blocs erratiques» $(1996: 22)$ : advienne que pourra, chercher noise, au fur et à mesure, etc. soit des séquences hétéro-entité: des noms formés à partir de verbes: un rendez-vous, un va-et-vient, un suivez-moi-jeune-bomme, des adjectifs formés à partir de syntagmes prépositionnels : une personne à la mode, un costume sur mesure, une idée dans le vent, une femme d'un certain âge, des séquences adverbiales ayant la même structure : à tâtons, en hâte, de guerre lasse, etc. Cette opposition recoupe celle des unités endocentriques et exocentriques ${ }^{1}$ : un nid de poule est exocentrique et auto-entité ; un vaurien est exocentrique et hétéro-entité ; une jeune fille est endocentrique et auto-entité ; seul le dernier cas de figure n'est pas possible : une unité endocentrique et hétéro-entité (si on ne tient pas compte des unités doublement figées). La notion de "degré de figement» qui correspond le mieux à la réalité du fonctionnement des séquences figées concerne de fait les unités dites «hétéro-entité » dont le prototype est la locution verbale.

Pour tenter de mesurer la gradation du figement, il ne faut pas limiter la notion de degré à l'unique dimension syntaxique. Une corrélation étroite avec le contenu sémantique fait que l'un va rarement sans l'autre: le dédoublement en tant que caractéristique des séquences polylexicales a pour conséquence que deux significations coexistent dans la même séquence, un sens global qui correspond à la totalité de la séquence et un sens littéral, sous-jacent, qui est réactivé en cas de besoin dans le discours. Par ailleurs, comme on peut parler de degré de figement syntaxique, on peut parler de degré d'opacité sémantique. Comparons les quatre dénominations suivantes : vin rouge, vin blanc et vin gris. Rien qu'en s'interrogeant sur la couleur du vin, on s'aperçoit que la rupture est gradable : le vin rouge est rouge, les vins blancs et gris ne sont ni blancs ni gris.

Découlent de cette situation les éléments suivants d'un projet de description :

- la description des séquences figées doit faire partie intégrante de la description de la totalité des items lexicaux de la langue ; le tout s’inscrit dans un continuum qui va du

\footnotetext{
${ }^{1}$ Rappelons que la première opposition repose sur la présence ou l'absence dans la séquence figée d'un élément renvoyant à l'entité dénommée (une jeune fille est une fille; un nid de poule n'est pas un nid. La seconde indique le transfert entre la catégorie de départ et la catégorie d'arrivée ; on parle d'autoentité quand la formation se conçoit dans la même catégorie grammaticale comme c'est le cas pour les locutions verbales, et d’hétéro-entité quand la séquence figée est versée dans une catégorie autre que celle dans laquelle elle a été conçue (un pince-sans-rire).
} 
moins contraint au plus contraint, du plus libre au moins libre. Toutes les contraintes décrites ne sont pas particulièrement propres aux séquences figées. L'exemple de la passivation illustre bien cette situation ;

- la description des séquences figées relève donc du programme général de la description de toute la langue. Que le prédicat soit polylexical ou monolexical, il obéit dans tous les cas de figure à un ensemble de contraintes qui représentent son emploi dans la langue ;

- pour mesurer la gradation dans le figement que connaissent les séquences polylexicales, il faut procéder à toutes les manipulations générales partagées par toutes les séquences (comme la commutation) et passer ensuite aux manipulations spécifiques à la partie du discours à laquelle appartient la séquence. C'est à partir du croisement des deux qu'il sera possible de mesurer le degré du figement de chaque séquence. Prenons les exemples suivants: (1) faire la pluie et le beau temps, (2) ne pas avoir inventé le fil à couper le beurre, (3) avoir avalé sa langue, (4) enterrer la bache de guerre. Si on leur applique un certain nombre de restructurations, nous constatons qu'ils n'ont pas le même comportement. Plus le nombre de contraintes est grand, plus les séquences sont figées :

$\begin{array}{lllll} & 1 & 2 & 3 & 4 \\ \text { passivation } & - & - & - & + \\ \text { négation } & + & \cdot & + & + \\ \text { contrainte aspect. } & - & + & + & -\end{array}$

etc.

Ce n'est que lorsqu'on intègre la description du figement dans le projet général de la description de la langue qu'on pourrait parler de traitement automatique.

\section{Figement et traitement automatique}

Trois difficultés majeures se dressent devant le traitement automatique des langues: la polysémie, le figement et l'inférence. L'analyse en classes d'objets était conçue initialement pour résoudre le problème de la polysémie. Les analyses que nous menons actuellement au LLI conduisent à l'analyse suivante :

- figement et classes d'objets

- si l'on part du principe qu'une unité polylexicale fonctionne comme l'unité monolexicale équivalente, il n'y a pas lieu de les dissocier dans la description générale ;

- les prédicats seraient classés selon qu'ils appartiennent à des actions, des états et des événements en leur appliquant les mêmes tests. Le fait de pouvoir reprendre ou non une locution par faire peut nous servir 
Salab Mejri

d'indication sur la nature du prédicat: Luc lui a tiré les vers du nez comme il le fait souvent avec les invités;

- comme toutes les autres unités lexicales, les séquences figées sélectionnent des arguments spécifiques: NO<bum> casser sa pipe, boire le bouillon d'onze heures, passer l'arme à gauche... ;

- le traitement en classes d'objets permet de voir dans les constructions à verbes supports des cas intermédiaires entre les locutions verbales et les constructions libres. Avec les verbes supports appropriés, on est à la limite du figement : commettre un crime, intimer l'ordre de, caresser l'espoir de...;

- les séquences adjectivales polylexicales se laissent décrire comme toutes les autres séquences en termes de prédicats et d'arguments : dans Luc est à la mode, on voit que la séquence à la mode, tout comme un adjectif simple comme élégant, admet pratiquement toute la syntaxe de l'adjectif : elle peut être attribut comme c'est le cas dans l'exemple cité, épithète (une femme à la mode), apposée (Luc, à la mode, ...), accepte l'intensif (elle est très à la mode) et la coordination avec des segments ayant la même valeur syntaxique ( $A$ la mode et peu soucieuse du regard des autres, elle...);

- les travaux menés dans le cadre du LLI sur la prédication du second ordre montrent qu'un très grand nombre de locutions comme dans le but de à valeur syntaxique se laissent décrire en termes de prédicats et d'arguments (G. Gross et M. Prandi 2004 : 157-160) :

. tirer an but, Paris était le but de notre voyage;

. dans le but de, mais également avec le but affiché de;

. dans ce but, dans un tel but, dans un but similaire, dans aucun but précis, dans nul autre but, dans quel but?

. Paul poursuit des buts contradictoires, ces deux buts étant atteints ;

Cette mesure doit être prise dans un but de clarté sinon d'apaisement;

. dans un but commercial, politique, militaire, utilitaire;

. aller droit au but.

\section{- figement et inférences}

- comme toutes les unités lexicales, les séquences figées s'inscrivent dans la structuration inférentielle du lexique (R. Martin 1976) : tourner autour du pot infère qu'on hésite à aller droit au but ou que ce qu'on cherche à exprimer pose quelques difficultés ;

- s'ajoute à ce type d'inférence une inférence particulière spécifique aux séquences figées : potentielle. Elle peut être réactivée selon les besoins du 
Figement absolu ou relatif: la notion de degré de figement

discours. Elle provient du dédoublement du sens des séquences (sens global et sens compositionnel) déjà évoqué et représente une source inépuisable de jeux dans le discours. Par une sorte d'héritage des propriétés des constituants se construit un discours qui implique à la fois les deux significations : leur météo devrait être une science du tonnerre; en définitive, c'est du vent $(\mathrm{R}$. Bacri) ;

- le figement peut être à l'origine d'une inférence fixée une fois pour toutes dans le lexique comme c'est le cas dans les proverbes: en plus de l'inférence assurée par les structures syntaxiques, nous avons dégagé dans le dernier colloque d'Europhras (août, Bâle 2004), une régularité lexicale que nous avons appelée métonymie inférentielle qui participe à la structuration des proverbes :

. Chose bien commencée est à demi achevée.

. L'exception confirme la règle.

. Mieux vaut tard que jamais.

. Qui aime bien châtie bien.

. Un de perdu, dix de retrouvés.

\section{Pour ne pas conclure}

Il est clair que le figement couvre la totalité des champs de la linguistique. En tant que phénomène général et systématique, il impose une relecture de toute la syntaxe, la sémantique et l'analyse du discours. Avec la notion de "degré de figement», il faut disposer d'une description détaillée pour chaque séquence. Les études actuelles s'orientent du côté de la constitution de grands corpus et de la description détaillée et minutieuse de tous les emplois. Cela se fait dans le cadre de la même langue ou en rapport avec la traduction, ce qui ouvre paradoxalement de grandes perspectives pour les langues de spécialité et appuie la thèse selon laquelle ces langues sont d'abord des langues auxquelles on applique la méthodologie descriptive appliquée à la langue générale (Lerat). 
Salab Mejri

\section{BIBLIOGRAPHIE}

Anscombre, J.-Cl. (2000), "Parole proverbiale et structures métriques », in Anscombre J.-Cl. (ed.), Langages 139, Paris, Larousse, pp. 6-26.

Anscombre, J.-Cl. (2003), «Les proverbes sont-ils des expressions figées ?», in Mejri S. (éd.) Cabiers de lexicologie 82, Paris, Champion, pp. 159-173.

BALly, Ch. (1951), Traité de stylistique française, Paris, Klincksieck.

Bonhomme, M. (1987), Linguistique de la métonymie, Berne, Peter Lang.

CAmugli Gallardo, C. (2003), "Qu'est-ce que tu chantes là ? Syntaxe et lexique dans le cadre des expressions métaphoriques figées », Cahiers de lexicologie 82, Paris, Champion, pp. 175192.

Damourette, J. et Pichon, E. (1968), Des mots à la pensée, Paris, édition d'Artrey.

DARMESTETER, A. et al. (1932, rééd. 1964), Dictionnaire général de la langue française du commencement du XV TIe siècle jusqu'à nos jours, précédé de Traité de la formation de la langue, Paris, Delagrave.

Fiala, P., Habert, B., Lafon, P., Pineira, C. (1978), « Des mots aux syntagmes. Figements et variation dans la Résolution du congrès de la CGT de 1978 », Mots 14, mars, pp. 47-88.

GaAtone, D. (1976), «Locutions prépositives et groupes prépositionnels », Linguistics 167, pp.15-33.

GaAtone, D. (1981), «Conjonctions et locutions conjonctives en français », Folia linguistica 14, pp. 195-211.

GonZalez Rey, I. (2002), La phraséologie du français, Toulouse, Presses Universitaires du Mirail.

GiRY-SCHNEIDER, J. (1986), "Les noms construits avec faire: compléments ou prédicats », Langue française 69, pp. 49-63.

GrÉCIANO, G. (1983), Signification et dénotation en allemand. La sémantique des expressions idiomatiques, Université de Metz.

GougenheIM, G. (1971), «Une catégorie lexico-grammaticale : les locutions verbales », Études de linguistique appliquée 2, pp. 56-64.

Gross, G. (1994), «Classes d'objets et description des verbes », Langages 115, pp.15-30.

Gross, G. (1996), Les expressions figées en français, Paris, Ophrys.

Gross, G. \& VivÈs, R. (2000), «La description des classes d'objets et l'enseignement des langues », Langue française 131 pp. 38-51.

Gross, G. et Prandi, M. (2004), La finalité. Fondements conceptuels et genèse linguistique, BruxellesParis, De Boeck, Duculot.

Gross, M. (1982), «Une classification des phrases «figées » du français », Revue québécoise de linguistique 11, 2, pp.151-185.

Gross, M. (1988a), «Les limites de la phrase figée », Langages 90, pp. 7-22. 
Figement absolu ou relatif: la notion de degré de figement

Gross, M. (1988b), «Sur les phrases figées complexes du français », Langue française 77, pp. 4770.

Grossmann, F. et Tutin, A. (eds.) (2003), Les collocations. Analyse et traitement. Travaux et recherches en linguistique appliquée, Amsterdam, Editions « De Werelt».

KLeIBER, G. (1999), Problèmes de sémantique. La polysémie en questions, Lille, Presses du Septentrion.

KLeIBER, G. (2000), «Sur le sens des proverbes », in Anscombre J.-Cl. (ed.), Langages 139, Paris, Larousse, pp. 39-58.

KLeiber, G. (2003), «Item lexical, mots construits et polylexicalité vus sous l'angle de la dénomination », in Mejri S. (ed.), Syntaxe et sémantique 5, Presses universitaires de Caen, pp. 31-46.

Le Pesant, D. et Mathieu-Colas, M. (1998), "Introduction aux classes d'objets », in Le Pesant D. \& Mathieu-Colas M. (eds), Langages 131, Paris, Larousse, pp. 6-33.

LERAT, P. (1995), Les langues spécialisées, Paris, PUF.

PICOCHE, J. (1993), Didactique du vocabulaire français, Paris, Nathan université.

MARTIN, R. (1976), Inférence, antonymie et paraphrase, Paris, Klincksieck.

MARTin, R. (2001), Sémantique et automate, Paris, PUF.

MEL'ČUK, I. (2003), «Les collocations : définition, rôle et utilité », in Grossmann F. et Tutin A. (eds.) pp. 23-31.

MEJRI, S. (1997), Le figement lexical. Descriptions linguistiques et restructuration sémantique, Tunis, Publications de la Faculté des lettres de la Manouba.

MEJRI, S. (1998a), « La conceptualisation dans les séquences figées », in Mejri S. (ed.), l'Information grammaticale, numéro spécial Tunisie, Paris, pp. 41-48.

MejRI, S. (1998b), «La globalisation sémantique », in Red. W. Banys. Katowice : Neophilologica, T 13, Pologne, pp. 83-93.

MEJRI, S. (2000), « Figement et dénomination », META 45, 4, pp. 609-621.

MEjRI, S. (ed.) (2003), Cabiers de lexicologie 82, Paris, Champion.

MejRI, S. (ed.) (2004a), Syntaxe et sémantique 5, Presses universitaires de Caen.

MEjRI, S. (2004b), « Polysémie et polylexicalité », in Mejri S. (ed.), Syntaxe et sémantique 5, Presses universitaires de Caen, pp. 13-30.

MejRI, S. (2004c), «L'idiomaticité : problématique théorique», in S. Mejri, T. Baccouche, H. Burger, Buhofer (eds), L'espace euro-méditerranéen : une idiomaticité partagée II, Tunis, CERES.

MEJRI, S. (à paraitre), «Structure inférentielle des proverbes », Colloque d'Europhras, Bâle, août 2004.

Mortureux, M-F. (2003), « Figement lexical et lexicalisation», Cabiers de lexicologie 82-2. 
Salab Mejri

Neveu, F. (2004), Dictionnaire des sciences du langage, Paris, Armand Colin.

Ruwet, N. (1983), «Du bon usage des expressions idiomatiques dans l'argumentation en syntaxe générative », Revue québécoise de linguistique 13, 1, pp. 9-145.

SCHMID, A.-M. (1992), "Mettre à toutes les sauces». Analyse sémantico-syntaxique des lexies complexes à base "mettre », Paris, Klincksieck, collection Recherches linguistiques XV.

SECHeHAYE, A. (1921), "Locutions et composés ", Journal de psychologie normale et pathologique, XVII, France, pp. 654-675.

TAmba, I. (2000), «Formules et dire proverbial », in Anscombre J.-Cl. (ed.), Langages 139, Paris, Larousse, pp.110-118.

Travaux du CERLICO (2001), Grammaticalisation 2 Concepts et cas, Presses universitaires de Rennes.

VIVÈS, R. (1998), " "Les mots pour le dire" : vers la constitution d'une classe de prédicats », Langages 131, pp. 64-76. 